\title{
Oropharyngeal dysphagia is a risk factor for community-acquired pneumonia in
} the elderly

\author{
Jordi Almirall*,\#,ף,**, Laia Rofes ${ }^{+, * *, \text { Mateu Serra-Prat }}{ }^{+, \S}$, Roser Icart*, \\ Elisabet Palomera ${ }^{\S}$, Viridiana Arreola ${ }^{f}$ and Pere Clavé ${ }^{\uparrow,+, f}$
}

ABSTRACT: The aim of this study was to explore whether oropharyngeal dysphagia is a risk factor for community-acquired pneumonia (CAP) in the elderly and to assess the physiology of deglutition of patients with pneumonia.

In the case-control study, 36 elderly patients (aged $\geqslant 70$ years) hospitalised with pneumonia were matched by age and sex with two independently living controls. All subjects were given the volume-viscosity swallow test to identify signs of oropharyngeal dysphagia. In the pathophysiological study, all cases and $\mathbf{1 0}$ healthy elderly subjects were examined using videofluoroscopy.

Prevalence of oropharyngeal dysphagia in the case-control study was $91.7 \%$ in cases and $40.3 \%$ in controls $(p<0.001)$. Adjusting for functionality and comorbidities, dysphagia showed an independent effect on pneumonia (OR 11.9, 95\% Cl 3.03-46.9). Among cases in the pathophysiological study, $16.7 \%$ showed safe swallow, $30.6 \%$ high penetrations, $36.1 \%$ severe penetrations and $16.7 \%$ silent aspirations during videofluoroscopy, while in the healthy elderly subjects these percentages were $80 \%, 20 \%, 0 \%$ and $0 \%$, respectively $(p<0.001)$. A delay in closure of the laryngeal vestibule $(0.414 \pm 0.029 \mathrm{~s}$ versus $0.200 \pm 0.059 \mathrm{~s}, \mathrm{p}<0.01)$ was the main mechanism of impaired airway protection.

In elderly subjects, oropharyngeal dysphagia is strongly associated with CAP, independently of functionality and comorbidities. Elderly patients with pneumonia presented a severe impairment of swallow and airway protection mechanisms. We recommend universal screening of dysphagia in older persons with pneumonia.

KEYWORDS: Ageing, aspiration pneumonia, pneumonia, swallowing

ommunity-acquired pneumonia (CAP) is a common disease and a frequent cause of hospitalisation and death among the elderly [1]. According to population-based studies, the annual incidence rate of CAP in adults varies between 2.6 and 13.4 per 1000 inhabitants, and is somewhat higher in elderly subjects $[2,3]$. Studies found old age to be a relevant risk factor for the acquisition of pneumonia [4]. Other associated factors that predispose to CAP in the elderly include: lifestyle and patient characteristics such as smoking, alcohol use, poor functional and nutritional status, weight loss and use of immunosuppressants; comorbidities such as heart disease, renal disease and chronic obstructive pulmonary disease (COPD); and environmental exposures such as second-hand smoke, gases, fumes and chemicals [5-7].
We recently found that oropharyngeal dysphagia was also a highly prevalent clinical finding in up to $23 \%$ of independently living elderly subjects (aged $>70$ years), $0.74 \%$ of them presenting signs of aspiration during swallow [8]. In these patients with oropharyngeal dysphagia, a decrease in the efficacy of deglutition was associated with development of malnutrition and a decrease in safety was associated with a high prevalence of respiratory infections during follow-up [9]. Oropharyngeal dysphagia has been identified as a serious risk factor for developing aspiration pneumonia in frail elderly people [10]. The pathogenesis of aspiration pneumonia in immunocompetent adults has been attributed to pharyngeal colonisation of respiratory pathogens and subsequent inhalation of infectious particles [11]. Oropharyngeal dysphagia has also been proposed

This article has supplementary material available from www.erj.ersjournals.com

AFFILIATIONS

*Intensive Care Unit, Hospital de Mataró, Consorci Sanitari del Maresme, Mataró

*Centro de Investigación Biomédica en Red de enfermedades respiratorias (CIBERES), Instituto de Salud Carlos III, Madrid, "Dept of Medicine and Surgery, Universitat Autònoma de Barcelona, Barcelona,

+Centro de Investigación Biomédica en Red de enfermedades hepáticas y digestivas (CIBEREHD), Instituto de Salud Carlos III, Madrid,

${ }^{\S}$ Research Unit, Hospital de Mataró, Consorci Sanitari del Maresme,

Mataró, and

${ }^{f}$ Gastrointestinal Motility Unit,

Hospital de Mataró, Consorci Sanitar del Maresme, Mataró, Spain.

${ }^{* \star B}$ Both authors contributed equally.

CORRESPONDENCE

J. Almirall

Intensive Care Unit

Hospital de Mataró

Carretera de Cirera s/n

08304 Mataró

Spain

E-mail: jalmirall@csdm.cat

Received:

Jan 312012

Accepted after revision:

July 022012

First published online:

July 262012 
as an independent risk factor associated with CAP in the elderly $[6,12,13]$, but this has not yet been proved. Moreover, the pathogenic mechanism that leads to oropharyngeal dysphagia in the frail elderly and in neurological patients has been identified in recent years [14, 15]. In contrast, the pathophysiology of impaired swallow response of elderly people with pneumonia has not yet been studied.

The present study has two main objectives: 1) to provide further evidence of the association between oropharyngeal dysphagia and CAP in the elderly, and 2) to assess the pathophysiology of oropharyngeal dysphagia in elderly subjects with CAP.

\section{METHODS}

\section{Case-control study}

An observational case-control study was designed. The study protocol was approved by the Ethics Committee of Consorci Sanitari del Maresme (Mataró, Spain). Cases were defined as subjects aged $\geqslant 70$ years with confirmed CAP that required hospitalisation in the Hospital of Mataró between February 2008 and February 2010 and were consecutively included in the study, as long as videofluoroscopic examination was available. We have described the criteria for the diagnosis of CAP previously [2]. Patients from nursing homes or those who had been discharged from hospital $\leqslant 7$ days before the onset of symptoms were excluded. For each case, two matched (by sex and age) controls without CAP were randomly selected from the list of independently living elderly subjects assigned to the Cirera-Molins Primary Care Centre in Mataró (Spain). All cases and controls were explored by the volume-viscosity swallow test (online supplementary material) to assess the clinical signs for oropharyngeal dysphagia and impaired efficacy and safety of swallow. Comorbidities and pre-admission functional capacity were also registered for all participants.

\section{Pathophysiological study}

Details of the clinical characteristics of pneumonia and concomitant medications were collected in all cases. Vaccination history, number of previous pneumonias, fever, days of clinical symptoms, lobes affected, severity of CAP [16], intensive care unit admissions and hospital death were recorded. To determine the aetiology of pneumonia, blood cultures $(n=30)$ and urine antigen tests for Streptococcus pneumoniae $(\mathrm{n}=30)$ and Legionella pneumophila $(\mathrm{n}=33)$ were performed. Respiratory samples were also obtained whenever possible, of sputum $(n=9)$, tracheal aspirate $(n=1)$ and pleural fluid $(n=2)$. A sublingual smear $(n=29)$ was also obtained to assess the oral flora.

10 healthy, elderly subjects (aged $\geqslant 70$ years) and all patients with CAP were studied by videofluoroscopy during admission. Details on the videofluorscopic procedures are provided in the online supplementary material. Digitisation and analysis of videofluoroscopic images were made using the software Swallowing Observer (Image and Physiology SL, Barcelona, Spain). Laryngeal vestibule penetrations and tracheobronchial aspirations were classified according to a validated scale [17] (online supplementary figure S1), and oropharyngeal residue was also identified. Timing of the oropharyngeal swallow response, hyoid bone movement and bolus velocity $\left(\mathrm{m} \cdot \mathrm{s}^{-1}\right)$ were also measured [14].
CAP patients were followed through the electronic clinical records of the hospital of Mataró for 1 year or contacted by telephone 1 year after discharge in order to register death or readmissions caused by lower respiratory tract infections. If telephone contact could not be made, the family doctors of the patients were contacted.

\section{Statistical analysis}

Categorical variables were described as percentages and compared by the Chi-squared test or the Fisher's exact test when appropriate. Quantitative variables were described as mean \pm SEM and compared by the Mann-Whitney U-test. As a measure of association between oropharyngeal dysphagia, impaired efficacy and safety of swallow and pneumonia, estimations of the relative risk through odds ratios and $95 \%$ confidence intervals were calculated. The effect of oropharyngeal dysphagia and impaired safety and efficacy of swallow on the risk of the development of CAP was adjusted by comorbidities and pre-admission functional capacity in a multivariate model using logistic regression. Survival curves, according to safety of swallow, were compared using a logrank test. Statistical significance was accepted if p-values were $<0.05$. Statistical analysis was performed using SPSS 15.0 (SPSS Inc., Chicago, IL, USA).

\section{RESULTS}

\section{Case-control study}

45 cases with CAP were screened during the study period and nine cases were excluded because patients were discharged before the videofluoroscopic examination was available. 36 cases with CAP (81.22 \pm 0.77 years, 24 males) were finally included in the study and matched with 72 controls $(81.21 \pm 0.53$ years, 48 males). The univariate analysis showed that the prevalence of oropharyngeal dysphagia and clinical signs of impaired efficacy and safety of swallow were higher in CAP cases than in controls (table 1). CAP cases also presented lower functional capacity than controls, according to the Barthel index (67.1 versus 97.4, $p<0.001$ ), as well as higher prevalence of chronic bronchitis/ COPD and chronic heart failure. A multivariate logistic regression analysis showed an independent effect of oropharyngeal dysphagia related to the development of CAP when adjusting for suboptimal Barthel index scores $(<100)$, chronic bronchitis/ COPD, and chronic heart failure (table 2).

\section{Pathophysiological study}

General characteristics and aetiology of pneumonia

General features of patients with pneumonia are described in table 3. Most of them were treated with corticosteroids $(n=20$, $55.6 \%)$ and $\beta_{2}$-agonists $(n=20,55.6 \%) ; 15(41.7 \%)$ received proton-pump inhibitors; $13(36.1 \%)$ received diuretics and 12 $(33.3 \%)$ received angiotensin-converting enzyme inhibitors. Distribution of drugs acting on the central nervous system was as follows: benzodiazepines $(n=10,27.8 \%)$, antidepressants $(n=6,16.7 \%)$, neuroleptics $(n=3,8.3 \%)$ and anti-parkinsons drugs $(n=3,8.3 \%)$. The aetiological diagnosis of pneumonia was achieved in 20 patients $(55.6 \%)$. S. pneumoniae was found in $17(47.2 \%)$ patients, of whom nine $(52.9 \%)$ presented impaired safety of swallow and eight $(47.1 \%)$ presented safe swallow. Pseudomonas aeruginosa $(2.8 \%)$, Klebsiella penumoniae $(2.8 \%)$ and co-infection of $P$. aeruginosa and $K$. penumoniae $(2.8 \%)$ were also found as aetiologic agents in patients with 


\begin{tabular}{|c|c|c|c|c|}
\hline \multirow[t]{2}{*}{ TABLE 1} & \multirow[b]{2}{*}{ Cases } & \multirow[b]{2}{*}{ Controls } & \multirow[b]{2}{*}{$\mathrm{p}$-value } & \multirow[b]{2}{*}{ OR (95\% Cl) } \\
\hline & & & & \\
\hline Subjects & 36 & 71 & & \\
\hline Barthel index $<100$ & $25(69.4)$ & $18(25.0)$ & $<0.001$ & $6.82(2.81-16.6)$ \\
\hline $\begin{array}{l}\text { COPD/chronic } \\
\text { bronchitis }\end{array}$ & $18(50)$ & $20(27.8)$ & 0.023 & $2.60(1.13-5.98)$ \\
\hline Chronic heart failure & $16(44.4)$ & $15(21.1)$ & 0.012 & $2.99(1.25-7.13)$ \\
\hline Benzodiazepine use & $10(27.8)$ & $16(22.2)$ & 0.254 & $1.34(0.54-3.37)$ \\
\hline $\begin{array}{l}\text { Oropharyngeal } \\
\text { dysphagia }\end{array}$ & $33(91.7)$ & $29(40.3)$ & $<0.001$ & $16.3(4.57-58.2)$ \\
\hline Safety impairment & $22(61.1)$ & $18(25.0)$ & $<0.001$ & $4.71(2.00-11.1)$ \\
\hline Efficacy impairment & 30 (83.3) & $24(33.3)$ & $<0.001$ & $10.0(3.66-27.3)$ \\
\hline
\end{tabular}

impaired safety of swallow. In contrast, normal oral flora was found in $69.0 \%$ of oral smears. Candida albicans $(8.3 \%), P$. aeruginosa $(8.3 \%)$, K. penumoniae $(2.8 \%)$ and S. agalactiae $(2.8 \%)$ were the pathogens isolated from the oral cavities of patients.

\section{Videofluoroscopic study}

Efficacious swallows without any residue during all series of the videofluoroscopic study were observed in $60 \%$ of healthy elderly subjects $(75.80 \pm 0.97$ years, $70 \%$ male) and in $41.67 \%$ of elderly patients with CAP $(p=0.066)$ (fig. 1). Safe swallows (score 1 on the penetration-aspiration scale) were observed in $80 \%$ of healthy elderly subjects, and high penetrations into the laryngeal vestibule (score 2) in $20 \%$. In contrast, $16.7 \%$ of elderly patients with CAP showed safe swallow $(\mathrm{p}<0.001)$, $30.6 \%$ presented high penetrations, $36.1 \%$ severe penetrations into the laryngeal vestibule (not ejected from the airways and/ or contacting the vocal folds, scores 3-5) and $16.7 \%$ silent aspirations (score 8) (fig. 1). General prevalence of oropharyngeal dysphagia among CAP patients according to the videofluoroscopic study was $75.0 \%$. In healthy elderly subjects, total duration of swallow response (time from glossopalatal junction opening to laryngeal vestibule opening) for 5-mL nectar boluses was $0.888 \pm 0.042 \mathrm{~s}$, the interval for oropharyngeal reconfiguration from a respiratory to a digestive pathway (time to laryngeal vestibule closure) was $0.240 \pm 0.039 \mathrm{~s}$ and timing of upper oesophageal sphincter opening was $0.276 \pm 0.039$ s. Elderly patients with CAP showed similar duration of swallow response $(1.00 \pm 0.042 \mathrm{~s} ; \mathrm{p}=0.241)$ and time to upper oesophageal sphincter opening $(0.333 \pm 0.025 \mathrm{~s} ; \mathrm{p}=0.178)$ as healthy elderly subjects, but demonstrated a significant delay in laryngeal vestibule closure $(0.414 \pm 0.029 \mathrm{~s}, \mathrm{p}=0.002)$. When comparing elderly patients with $\mathrm{CAP}$, according to the safety of swallow, patients with safe swallow (scores 1-2 on the penetration-aspiration scale) showed a significantly shorter laryngeal vestibule closure time than patients with impaired safety of swallow (scores 3-8) (fig. 2). In contrast, no differences were found in the timing of upper oesophageal sphincter opening $(0.287 \pm 0.024 \mathrm{~s}$ and $0.375 \pm 0.040 \mathrm{~s}$, respectively; $\mathrm{p}=0.144)$ or the total duration of swallow response $(0.948 \pm 0.039 \mathrm{~s}$ and $1.05 \pm 0.071 \mathrm{~s}$, respectively; $\mathrm{p}=0.437$ ). Regarding hyoid bone movement, healthy elderly
TABLE 2 Multivariate logistic regression analysis of risk factors associated with pneumonia in the elderly

\begin{tabular}{lcc} 
& p-value & OR (95\% CI) \\
\hline Barthel index $<100$ & 0.001 & $6.93(2.13-22.5)$ \\
COPD/chronic bronchitis & 0.032 & $3.80(1.12-12.9)$ \\
Chronic heart failure & 0.184 & $2.19(0.69-6.95)$ \\
Oropharyngeal dysphagia & $<0.001$ & $11.9(3.03-46.9)$ \\
\hline
\end{tabular}

COPD: chronic obstructive pulmonary disease.

subjects achieved maximal vertical extension in $0.314 \pm 0.041 \mathrm{~s}$ and maximal anterior extension in $0.349 \pm 0.058 \mathrm{~s}$. Elderly patients with CAP reached maximal vertical and anterior extension significantly later $(0.437 \pm 0.039 \mathrm{~s}$ and $0.549 \pm 0.040 \mathrm{~s}$, respectively; $\mathrm{p}<0.05)$. CAP patients with impaired safety of swallow also achieved maximal vertical extension later than CAP patients with safe swallow (fig. 3). Maximal vertical and anterior hyoid displacement was similar among groups (data not shown). Finally, the maximal velocity acquired by the bolus at the upper oesophageal sphincter was very similar between healthy elderly subjects $\left(0.481 \pm 0.073 \mathrm{~m} \cdot \mathrm{s}^{-1}\right)$ and elderly patients with CAP

$\begin{array}{ll}\text { TABLE } 3 & \begin{array}{l}\text { Clinical characteristics of patients with } \\ \text { pneumonia }\end{array}\end{array}$

\begin{tabular}{lc} 
Subjects $\mathbf{n}$ & 36 \\
Influenza vaccination & $20(55.6)$ \\
Pneumococcal vaccination & $5(13.9)$ \\
Number of previous pneumonias & \\
$\quad 0$ & $25(69.4)$ \\
1 & $8(22.2)$ \\
$\geqslant 2$ & $3(8.3)$ \\
Body temperature $<^{\mathbf{3} 7^{\circ} \mathbf{C}}$ & $11(30.6)$ \\
Duration of clinical symptoms days & $3.9 \pm 3.6$ \\
Lung location & \\
RUL & $14(38.9)$ \\
RLL & $11(30.6)$ \\
ML & $3(8.3)$ \\
LUL & $3(8.3)$ \\
LLL & $11(30.6)$ \\
$\geqslant 2$ affected lobes & $6(16.7)$ \\
PSI & \\
I & 0 \\
II & 0 \\
III & \\
IV & $8(22.2)$ \\
V & $21(58.3)$ \\
CRB-65 score & $7(19.4)$ \\
ICU admission & $2.0 \pm 0.9$ \\
Hospital death & $7(19.4)$ \\
& $3(9.7)$ \\
\hline
\end{tabular}

Data presented as $\mathrm{n}(\%)$ or mean $\pm \mathrm{SD}$, unless otherwise stated. RUL: right upper lobe; RLL: right lower lobe; ML: middle lobe; LUL: left upper lobe; LLL: left lower lobe; PSI: pneumonia severity index; CRB-65: confusion, respiratory rate $\geqslant 30$ breaths $\cdot \mathrm{min}^{-1}$, systolic blood pressure $<90 \mathrm{mmHg}$ or diastolic blood pressure $\leqslant 60 \mathrm{mmHg}$, age $\geqslant 65$ years; ICU: intensive care unit. 

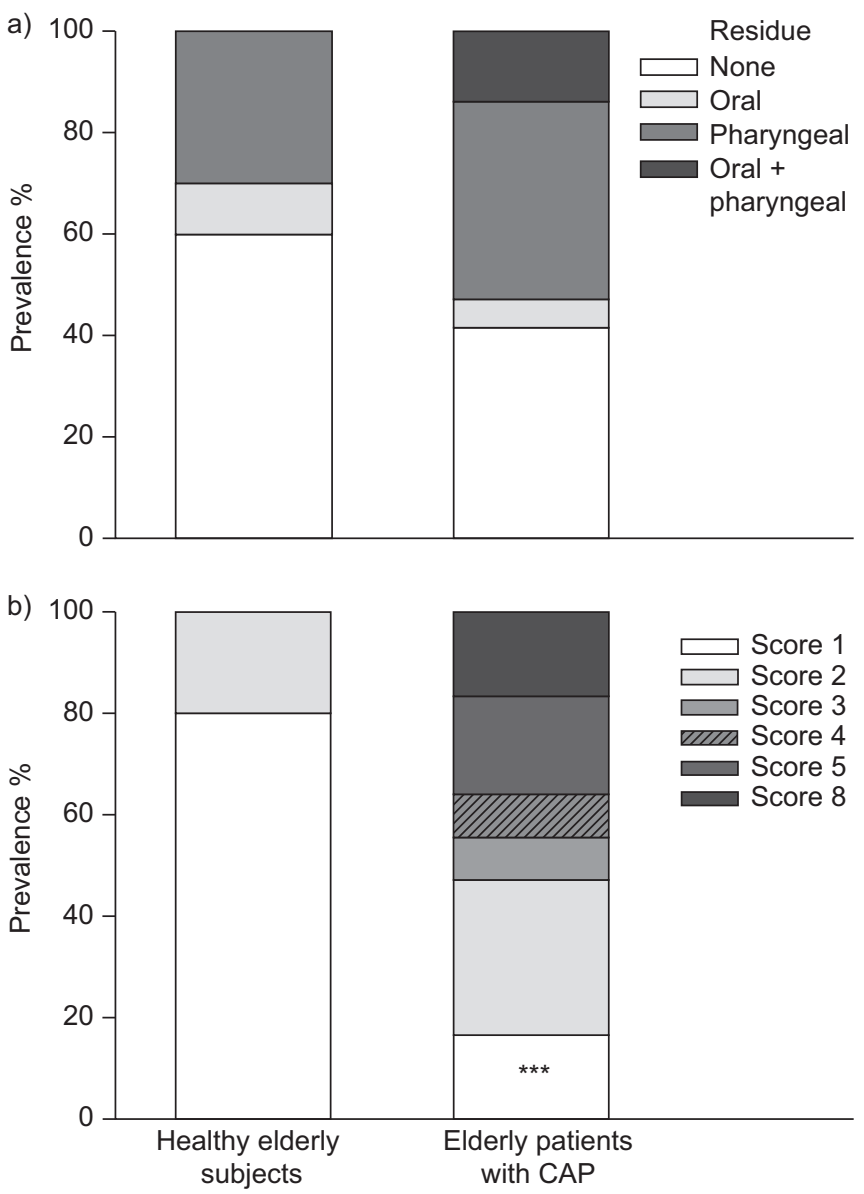

FIGURE 1. Prevalence of main videofluoroscopic signs of a) efficacy (oropharyngeal residue) and b) safety of swallow (penetration-aspiration scale). Prevalence is expressed as number of subjects with sign presence versus total number of subjects. CAP: community-acquired pneumonia. ${ }^{* *}$ : $p<0.001$.

$\left(0.482 \pm 0.035 \mathrm{~m} \cdot \mathrm{s}^{-1} ; \mathrm{p}=0.946\right)$ and was not affected by impairment in the safety of swallow.

\section{1-year follow-up}

CAP patients with impaired safety of swallow showed decreased survival rates compared to patients with safe swallow 1 year after admission (fig. 4 ). $\leqslant 50 \%$ of CAP patients with safe swallow and $71.43 \%$ with impaired safety of swallow $(p=0.201)$ were readmitted during the follow-up with lower respiratory tract infections.

\section{DISCUSSION}

The most remarkable finding of our study was that oropharyngeal dysphagia is strongly associated with CAP and should be considered as an independent risk factor for CAP in the elderly. The clinical assessment of oropharyngeal dysphagia showed that prevalence of dysphagia and impaired efficacy and safety of swallow among elderly patients with CAP was very high compared to matched controls. These clinical results were confirmed by the gold standard for swallowing evaluation, videofluoroscopy. The videofluoroscopic study showed that $52.8 \%$ of elderly patients with CAP presented severe penetrations or aspirations during swallow. We also observed

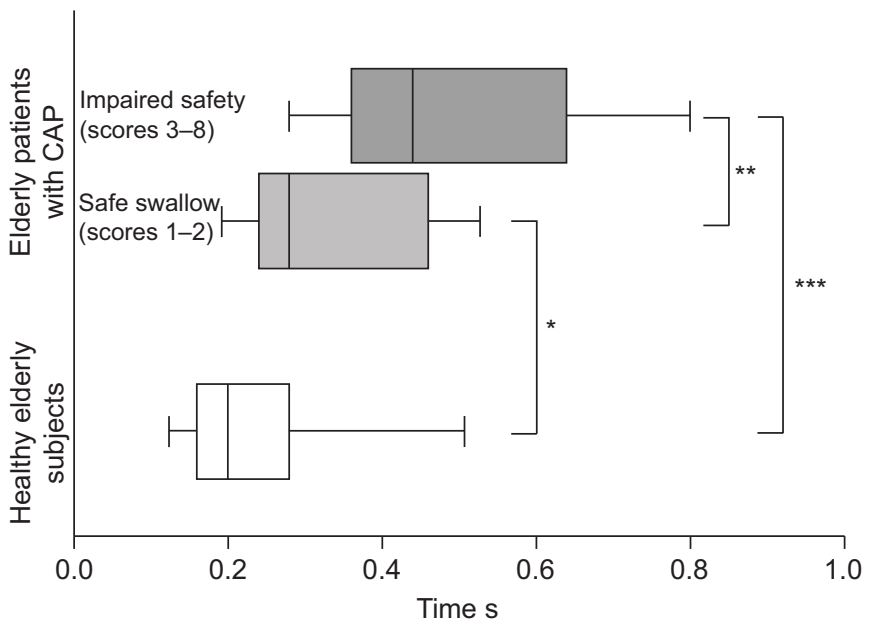

FIGURE 2. Laryngeal vestibule closure time. Patients with community-acquired pneumonia (CAP) were divided into patients with safe swallow (scores 1-2) and patients with impaired safety of swallow (scores 3-8). Each box plot represents the median, 10th, 25th, 75th and 90th percentiles. ${ }^{*}: p<0.05 ;{ }^{*}: p<0.01 ;{ }^{* *}: p<0.001$.

a high prevalence of oropharyngeal residue in these patients. Oropharyngeal residue is also an important finding, as it can predispose to oral colonisation and post-swallow aspirations [18]. Finally, the oropharyngeal swallow response was severely impaired in our elderly patients with CAP. A severe delay in the oropharyngeal reconfiguration from a respiratory phase to a digestive phase, caused by slow laryngeal vestibule closure and vertical hyoid motion, was the key parameter associated with impaired safety of swallow. Diagnostic aetiology of pneumonia was achieved in half of the cases. All patients with CAP and safe swallow presented S. pneumoniae as the aetiologic agent of pneumonia. Most patients with impaired safety of swallow also presented S. pneumoniae as the aetiologic agent, followed by Gram-negative bacilli. Taken together, our data strongly suggest that aspiration among patients with CAP is much more common than was believed and is a relevant pathogenic mechanism for elderly patients with pneumococcal $\mathrm{CAP}$, as well as for those with Gram-negative pneumonia.

Some studies have discussed the importance of aspiration and swallowing impairment in developing CAP $[6,12]$ in elderly patients, but, as far as we know, the present study is the first to evaluate the specific role of dysphagia as a predisposing and prognostic factor for CAP in the elderly and to assess the mechanisms of the impaired swallow response of elderly patients with CAP. Moreover, the assessment of dysphagia was made prospectively, both in cases and controls. The prospective evaluation of dysphagia is a relevant point because dysphagia is often underdiagnosed and several cases may be missed in clinical records. We used an accurate and validated clinical method for the clinical assessment of dysphagia, the volume-viscosity swallow test, and found that not only impaired safety of swallow is associated with CAP, but impaired efficacy of swallow is also a risk factor for CAP in the elderly. In healthy people, the effective clearance of most of pathogens from the oropharynx is due to effective salivary flow and efficient swallowing. Therefore, when the efficacy of swallow is impaired, a reduction in mechanical clearance occurs leading to oropharyngeal residue, and potential 

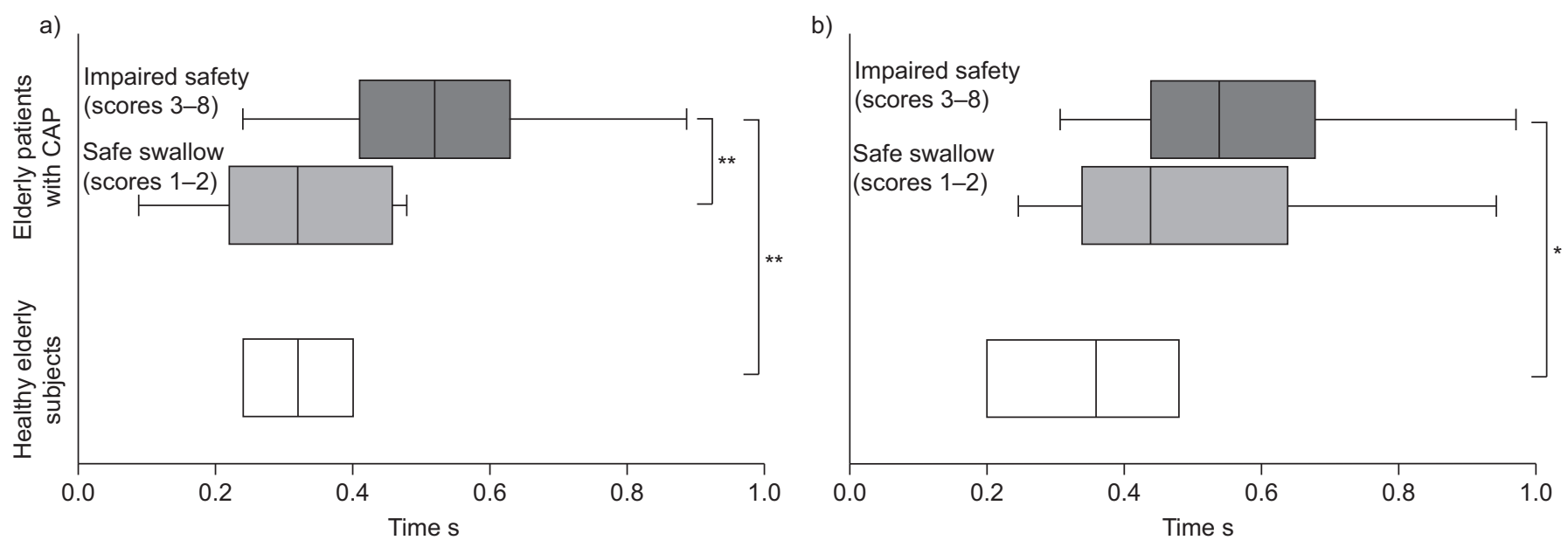

FIGURE 3. Hyoid movement. Time of maximal hyoid a) vertical and b) anterior extension of healthy elderly subjects and patients with community-acquired pneumonia (CAP) divided into patients with safe swallow (scores 1-2) and patients with impaired safety of swallow (scores 3-8). For elderly patients with CAP the box plot graphs show median, 10th, 25th, 75th and 90th percentiles. For healthy elderly subjects the box plot graphs show median, 25th and 75 th percentiles. ${ }^{*}: p<0.05,{ }^{*}: p<0.01$.

pulmonary or oropharyngeal pathogens may colonise the oropharynx and be a potential source of pulmonary infections [19]. TERAMOTO et al. [20] reported that the prevalence of aspiration pneumonia in patients with CAP aged $\geqslant 70$ years was $60.1 \%$, using a wide variety of clinical assessment methods. We found a prevalence of oropharyngeal dysphagia among patients with CAP of $91.7 \%$ by means of the volume-viscosity swallow test and of $75 \%$ in the videofluoroscopic study, with $52.8 \%$ of the patients having severe penetrations or aspirations during swallow. This is the first time, to the authors' knowledge, that a gold standard, videofluoroscopy, has been used to assess the prevalence of aspiration during swallow in elderly patients admitted with CAP. Prevalence of silent aspirations in our study was $16.7 \%$. A previous study, using a radioactive tracer, found that the occurrence of silent aspiration during sleep was $71 \%$ in CAP patients, whereas only $10 \%$ of agematched controls aspirated [21]. The aspiration of small amounts

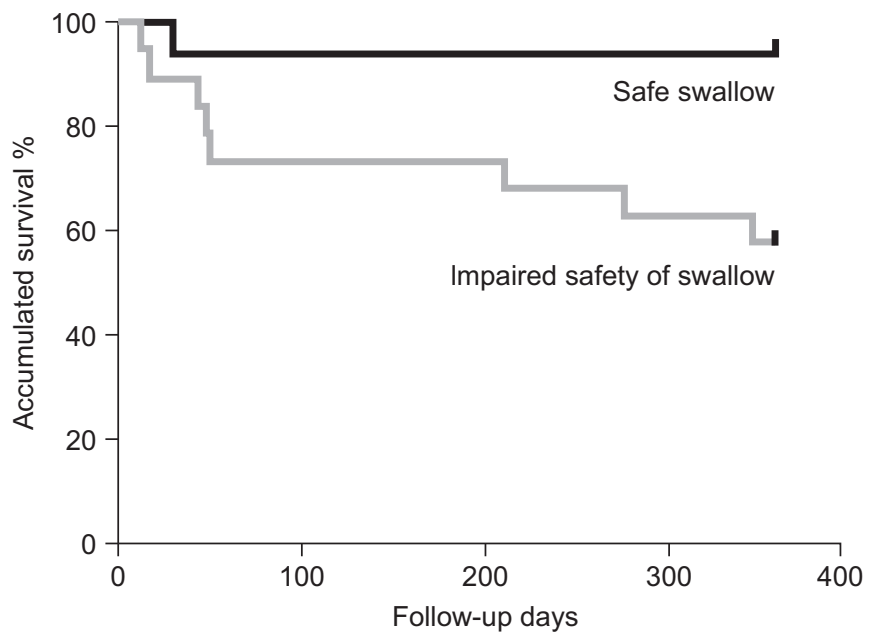

FIGURE 4. Survival curves. Accumulated survival of patients with communityacquired pneumonia at 1 year according to the impairment of swallow (safe swallow: scores 1-2 and impaired safety of swallow: scores 3-8). $p=0.0157$. of oropharyngeal secretions during sleep is a common finding in older adults $[22,23]$ and usually happens without consequences due to preserved cough reflex, ciliary transport and immune system and to the low virulence of normal pharyngeal flora. However, the silent aspiration of large amounts during swallowing is an abnormal and serious finding that, together with several factors common in the elderly such as progressive decrease in pulmonary function, decline in host defences, impaired cough reflex and increased oropharyngeal colonisation with respiratory pathogens can lead to aspiration pneumonia [19]. A key point of our study is that we elucidated the pathogenic mechanism of the aspiration process and impaired airway protection of patients with CAP through videofluoroscopy. Delayed airway protection caused by delayed laryngeal vestibule closure and vertical hyoid movement caused the aspirations and penetrations in elderly CAP patients. This impairment in swallow response is similar to that found in frail elderly patients [14] and patients with neurological diseases [15], and must be treated.

Our results show that impaired safety of swallow is a prognostic factor of mortality in elderly patients with CAP. These results agree with that found by RIQUELME et al. [12] and with one of our previous studies in patients with pneumonia admitted to an acute geriatric unit [24]. Again, the use of videofluoroscopy to determine patients with a predisposition to aspiration improves the diagnosis of aspiration pneumonia and confirms the results found by these previous studies using clinical records or clinical methods of screening.

A limitation of our study is that swallowing ability was not reevaluated after discharge to assess whether these patients had improved after the acute condition and also those who had deteriorated over time. Therefore, our design cannot exclude $\mathrm{CAP}$ as the cause of the swallowing problem. Another limitation was the small sample size. It was large enough to determine dysphagia as a risk factor for $\mathrm{CAP}$, to characterise the swallow response of CAP patients and to evaluate the impairment of swallow response as a prognostic factor. However, the small size limits the microbiological and clinical 
comparisons between CAP patients with and without oropharyngeal dysphagia. Further studies are needed in this area, including the assessment of oral health status and the use of molecular strategies to improve the assessment of oral microbiota in these patients. Conversely, although cases and controls were matched by age and sex, they could not be strictly comparable in terms of comorbidities, functional capacity or frailty. However, the multivariate analysis adjusted for the factors mentioned showed an independent effect of oropharyngeal dysphagia.

In summary, we have confirmed that oropharyngeal dysphagia is a major risk and prognostic factor for CAP in the elderly. We have established its high prevalence by means of a gold standard and determined that the pathogenic mechanism of impairment of swallow response in this cohort of patients with CAP was delayed airway protection. Therefore, we propose universal screening for oropharyngeal dysphagia in elderly patients admitted with CAP and the adoption of strategies to assess and treat oropharyngeal dysphagia when aspiration is suspected.

\section{SUPPORT STATEMENT}

This work was supported by grants from the Spanish Ministerio de Ciencia e Innovación (PI080448, PS09/01012 and INT 10/228) and the Agencia de Gestió d'Ajuts Universitaris i de Recerca (2009 SGR 708).

\section{STATEMENT OF INTEREST}

None declared.

\section{ACKNOWLEDGEMENTS}

We would like to thank Goretti Sauca (Dept of Microbiology, Hospital de Mataró, Mataró, Spain) for the microbiological assessments, Thaïs Zaro and Ivana Anglada (ICU, Hospital de Mataró, Mataró, Spain) for their help in the samples collection, Alberto Martin for his help in the videofluoroscopic studies and Jane Lewis (Gastrointestinal Motility Unit, Hospital de Mataró, Mataró, Spain) for her writing assistance.

\section{REFERENCES}

1 Jackson ML, Neuzil KM, Thompson WW, et al. The burden of community-acquired pneumonia in seniors: results of a population-based study. Clin Infect Dis 2004; 39: 1642-1650.

2 Almirall J, Bolibar I, Vidal J, et al. Epidemiology of communityacquired pneumonia in adults: a population-based study. Eur Respir J 2000; 15: 757-763.

3 Jokinen C, Heiskanen L, Juvonen $\mathrm{H}$, et al. Incidence of communityacquired pneumonia in the population of four municipalities in eastern Finland. Am J Epidemiol 1993; 137: 977-988.

4 Loeb M, McGeer A, McArthur M, et al. Risk factors for pneumonia and other lower respiratory tract infections in elderly residents of long-term care facilities. Arch Intern Med 1999; 159: 2058-2064.

5 Jackson ML, Nelson JC, Jackson LA. Risk factors for communityacquired pneumonia in immunocompetent seniors. J Am Geriatr Soc 2009; 57: 882-888.
6 Loeb M, Neupane B, Walter SD, et al. Environmental risk factors for community-acquired pneumonia hospitalization in older adults. J Am Geriatr Soc 2009; 57: 1036-1040.

7 Koivula I, Sten M, Makela PH. Risk factors for pneumonia in the elderly. Am J Med 1994; 96: 313-320.

8 Serra-Prat M, Hinojosa G, López D, et al. Prevalence of oropharyngeal dysphagia and impaired safety and efficacy of swallow in independently living older persons. J Am Geriatr Soc 2011; 59: 186-187.

9 Serra-Prat M, Palomera M, Gomez C, et al. Oropharyngeal dysphagia as a risk factor for malnutrition and lower respiratory tract infection in independently living older persons: a populationbased prospective study. Age Ageing 2012; 41: 376-381.

10 van der Maarel-Wierink CD, Vanobbergen JNO, Bronkhorst EM, et al. Meta-analysis of dysphagia and aspiration pneumonia in frail elders. J Dent Res 2011; 90: 1398-1404.

11 Marik PE. Aspiration pneumonitis and aspiration pneumonia. N Engl J Med 2001; 344: 665-671.

12 Riquelme R, Torres A, El-Ebiary $\mathrm{M}$, et al. Community-acquired pneumonia in the elderly: a multivariate analysis of risk and prognostic factors. Am J Respir Crit Care Med 1996; 154: 1450-1455.

13 Marik PE, Kaplan D. Aspiration pneumonia and dysphagia in the elderly. Chest 2003; 124: 328-336.

14 Rofes L, Arreola V, Romea M, et al. Pathophysiology of oropharyngeal dysphagia in the frail elderly. Neurogastroenterol Motil 2010; 22: 851-858.

15 Clavé $\mathrm{P}$, de Kraa M, Arreola V, et al. The effect of bolus viscosity on swallowing function in neurogenic dysphagia. Aliment Pharmacol Ther 2006; 24: 1385-1394.

16 Mandell LA, Wunderink RG, Anzueto A, et al. Infectious Diseases Society of America/American Thoracic Society consensus guidelines on the management of community-acquired pneumonia in adults. Clin Infect Dis 2007; 44: Suppl. 2, S27-S72.

17 Rosenbek JC, Robbins JA, Roecker EB, et al. A penetrationaspiration scale. Dysphagia 1996; 11: 93-98.

18 Gosney M, Punekar S, Playfer JR, et al. The incidence of oral Gramnegative bacteria in patients with Parkinson's disease. Eur J Intern Med 2003; 14: 484-487.

19 Palmer LB, Albulak K, Fields S, et al. Oral clearance and pathogenic oropharyngeal colonization in the elderly. Am J Respir Crit Care Med 2001; 164: 464-468.

20 Teramoto S, Fukuchi Y, Sasaki H, et al. High incidence of aspiration pneumonia in community- and hospital-acquired pneumonia in hospitalized patients: a multicenter, prospective study in Japan. J Am Geriatr Soc 2008; 56: 577-579.

21 Kikuchi R, Watabe N, Konno $\mathrm{T}$, et al. High incidence of silent aspiration in elderly patients with community-acquired pneumonia. Am J Respir Crit Care Med 1994; 150: 251-253.

22 Huxley EJ, Viroslav J, Gray WR, et al. Pharyngeal aspiration in normal adults and patients with depressed consciousness. Am J Med 1978; 64: 564-568.

23 Gleeson K, Eggli DF, Maxwell SL. Quantitative aspiration during sleep in normal subjects. Chest 1997; 111: 1266-1272.

24 Cabre M, Serra-Prat M, Palomera E, et al. Prevalence and prognostic implications of dysphagia in elderly patients with pneumonia. Age Ageing 2010; 39: 39-45. 